\title{
Vulnerabilidades, movilidades y corporalidades en jóvenes que realizan prácticas artísticas y deportivas en barrios populares de Buenos Aires
}

| ${ }^{1}$ Silvia Alejandra Tapia |

Resumen: El artículo presenta resultados de la tesis doctoral en la que se analizaron las experiencias de jóvenes de barrios populares que realizan prácticas artísticas y deportivas en la Ciudad Autónoma de Buenos Aires, indagando las vinculaciones entre cuerpos, emociones y movilidades espaciales en sus procesos de individuación. Aquí el análisis se centra en la lectura de las relaciones y tensiones entre las pruebas sociales que enfrentan estos jóvenes, y los soportes para atravesarlas, a partir de la utilización de la categoría de geometría de las vulnerabilidades, surgida de la articulación de las nociones de vulnerabilidad y geometría de los cuerpos. Para la construcción de los datos empíricos realicé entrevistas semi-estructuradas, relatos biográficos y participaciónobservación en una organización social que ofrece talleres gratuitos en dichos barrios. Este abordaje permitió advertir la dinámica y multidimensionalidad de los procesos de vulnerabilidad - donde las vinculaciones entre cuerposemociones-movilidades pusieron de manifiesto formas particulares y novedosas de dichos procesos y de soportes para afrontarlos - que atraviesan las experiencias de jóvenes de barrios populares.

> Palabras clave: juventudes; vulnerabilidades; movilidades; cuerpos; emociones.
1 Instituto de Investigaciones Gino Germani, Universidad de Buenos Aires/CONICET. Buenos Aires, Argentina (tapiasilvi1@gmail.com). ORCID: 0000-0003-0106-7023.

Recebido em: 01/08/2017 Revisado em: 12/06/2018 Aprovado em: 22/06/2018 


\section{Introducción}

En este trabajo presento resultados de la tesis doctoral ${ }^{1}$ en la que se analizaron las experiencias de jóvenes de sectores populares que realizan prácticas artísticas y deportivas en la Ciudad Autónoma de Buenos Aires (CABA), indagando las vinculaciones entre cuerpos, emociones y movilidades espaciales en sus procesos de individuación. La propuesta analítica se centró en la articulación de las herramientas conceptuales provenientes de tres corpus teóricos: la sociología de la individuación desarrollada por Danilo Martuccelli (2006; 2007); la sociología de los cuerpos/ emociones propuesta por Adrián Scribano (2012) y los estudios sobre movilidades espaciales, especialmente, a partir de los aportes de Tim Cresswell (2010).

De acuerdo con lo señalado por Pablo Di Leo y Ana Clara Camarotti (2013; 2015), a la luz de las transformaciones estructurales ocurridas en las últimas décadas por efecto de las políticas neoliberales, se evidenciaron desigualdades e inequidades sociales, territoriales, económicas, de género, étnicas, en las experiencias de $\operatorname{los}^{2}$ jóvenes de nuestra región. Esto interpeló la generación de nuevos enfoques que dieran un lugar significativo a las construcciones identitarias, las reflexividades y las experiencias singulares en el marco de los estudios sociales.

En ese sentido, la sociología de la individuación se posiciona como un abordaje particular que propone "reconstruir el carácter específico de una sociedad histórica a escala de sus individuos" (ARAUJO; MARTUCCELLI, 2012, p. 15). En una dialéctica entre lo común y lo singular, se rastrean las maneras en que se inscriben los procesos estructurales y los cambios históricos en las trayectorias individuales (MARTUCCELLI, 2007). La noción de prueba como operador analítico permite comprender el modo en que dichos procesos producen individuos. Desde una dimensión narrativa se reconstruyen las maneras en que éstos son percibidos como desafíos que los actores se ven forzados a enfrentar, aunque no de manera uniforme.

Para atravesar tales pruebas, los individuos requieren de soportes externos a ellos. Los soportes son los medios por los cuales el individuo puede sostenerse en el mundo; el conjunto de elementos, materiales e inmateriales, que lo vinculan a su contexto. Su análisis supone, no una mera enumeración de recursos disponibles, sino la identificación de las diversas maneras en que son movilizados por los individuos, aún enfrentando pruebas comunes (MARTUCCELLI, 2007; DI LEO; CAMAROTTI, 2013). 
Comprender los procesos de individuación de los jóvenes de barrios populares en el contexto de las sociedades latinoamericanas implica considerar la lógica capitalista que las rige, estableciendo modos correctos de sentir, de ser, de moverse - así como fugas y prácticas intersticiales que los resisten - para evitar y disminuir la conflictividad social que le permite sostenerse y reproducirse. La propuesta de la sociología de los cuerpos/emociones observa las particularidades de estas sociedades y el modo en que se establecen distancias y proximidades entre los cuerpos, que los marcan de manera desigual y plantean diferencias en la disponibilidad de sus energías y emociones (SCRIBANO, 2007; 2011; 2012; 2013).

Por, y a través, de los cuerpos es posible conocer el mundo y producir efectos en otros -actores, objetos, contextos - que estructuran las percepciones que organizan, naturalizando, las impresiones que se dan en los individuos que, a su vez, producen sensaciones a través de las que aquéllos distinguen un mundo como interno, subjetivo, y otro externo, social o natural. De esas sensaciones resultan las emociones, como la acción y el efecto de sentir o sentirse. En la interacción entre cuerpos y emociones, sus modos de regulación y sus potencialidades, se vuelve posible la comprensión de las limitaciones y posibilidades de acción y de movimiento (SCRIBANO, 2009; 2013).

Abordar la complejidad y diversidad de las prácticas de movilidad puede establecerse, siguiendo a Tim Cresswell (2010), a partir de la relación de tres aspectos: los movimientos físicos, es decir, aquéllos que permiten trasladarse de un lugar a otro; las representaciones y significaciones compartidas en torno a éstos, y las acciones de la vida cotidiana, actuadas y experimentadas a través del cuerpo. Prácticas codificadas social y culturalmente, valoradas diferencialmente según lo considerado moral, ético, estético, permitido o prohibido, en un marco de relaciones sociales en las que se entrecruzan condiciones como las de clase, género, etarias o étnicas. Dichas relaciones generadas por y en la movilidad suponen la producción de poder, poniendo de manifiesto el modo en que las movilidades son, al mismo tiempo, productoras de dichas relaciones y un producto de aquéllas.

En este artículo, se analiza el modo en que se intersectan y tensionan las dimensiones singulares y colectivas en las biografías de los jóvenes de sectores populares que realizan prácticas artísticas y deportivas, a partir de la utilización de la categoría de geometría de las vulnerabilidades. Primero, se describe la estrategia metodológica desarrollada y la propuesta de articulación de las nociones de vulnerabilidad (AYRES et al., 2008; DELOR; HUBERT, 2000) y geometría de los 
cuerpos (SCRIBANO, 2007) para la construcción de la noción de geometría de las vulnerabilidades como herramienta analítica. Luego, se presenta el análisis del corpus empírico en función de dos dimensiones: vulnerabilidades que generan distancias y vulnerabilidades en la proximidad. Dicho abordaje tiene como propósito advertir la dinámica y multidimensionalidad de los procesos de vulnerabilidad - donde las vinculaciones entre cuerpos-emociones-movilidades ponen de manifiesto formas particulares y novedosas de dichos procesos y de soportes para atravesarlos - que atraviesan las experiencias juveniles, problematizando las miradas estigmatizantes y homogeneizadoras acerca de los jóvenes de barrios populares.

\section{Abordaje metodológico}

Para la construcción de datos empíricos - siguiendo un enfoque cualitativo - se seleccionó el enfoque biográfico (LECLERC-OLIVE, 2009; DI LEO; CAMAROTTI, 2013). Se realizaron entrevistas semi-estructuradas, previo consentimiento informado, a 11 jóvenes (7 varones y 4 mujeres) con quienes se realizaron entre 3 y 7 encuentros (en total, 48 entrevistas). En dichas entrevistas, se identificaron acontecimientos significativos (LECLERC-OLIVE, 2009) que establecieran un giro existencial en la vida de estos jóvenes a partir de los cuales se construyeron, en forma consensuada, sus relatos biográficos. Fueron entrevistados jóvenes de entre 18 y 24 años que residían en barrios populares del Área Metropolitana de Buenos Aires (AMBA).

Por otra parte, siguiendo los aportes la etnografía, se realizó participaciónobservación (SCRIBANO, 2008). Con acuerdo de los coordinadores de una organización social (en adelante "el Circo") ${ }^{3}$ y de los jóvenes concurrentes, participé de talleres de Trapecio y tela, Acrobacia y malabares, Hip-hop y Tango, durante el segundo semestre de 2014. El Circo, desde 1998, ofrece talleres gratuitos en barrios del sur de la CABA, las zonas más vulnerabilizadas de la ciudad. Su propósito es contribuir a la transformación personal y comunitaria a través del arte. Promueven autodisciplina y creatividad, combinando juego y educación. Estimulan la convivencia, el reconocimiento de habilidades, responsabilidades y complementariedad con otros. Proponen una ruptura con los paradigmas asistencialistas que producen intervenciones desde una visión negativa y limitada de las juventudes, considerando, en cambio, sus potencialidades y capacidades. A 
su vez, procuran favorecer la accesibilidad generando dichos espacios en los barrios populares donde las ofertas artísticas y recreativas orientadas a los jóvenes resultan escasas (TAPIA, 2016).

En el caso de los entrevistados, todos residían en barrios populares del AMBA, pero sus situaciones habitacionales variaban significativamente. Algunos residían en viviendas propias - tomadas o cedidas por planes sociales - o habitaciones alquiladas. Otros contaban con viviendas familiares propias, en algunos casos, conviviendo más de una familia. Otros residían o habían permanecido en instituciones. Presentaban estructuras familiares diversas: cohabitación con padres y/o madres, hermanos, tíos y primos, parejas e hijos (sólo una de las jóvenes tenía hijos). Más de la mitad de sus padres y madres habían migrado desde países limítrofes o provincias del interior del país.

En cuanto a la escolaridad se presentaron diferencias significativas. Jóvenes con secundaria en curso, incluso en escuelas prestigiosas de la ciudad. Concurrencia a universidades públicas y privadas (en este caso, por medio de beca). Otros, en cambio, con situación de repitencia o abandono. La condición laboral también planteaba diversidad: trabajos informales y temporarios, ayuda en el cuidado de hermanos o negocios familiares y situación de desempleo con búsqueda activa.

Todos los entrevistados participaban, al menos, en uno de los talleres del circo. Habían accedido por su acercamiento directo al espacio o a partir de la relación con otros jóvenes concurrentes, pero mayormente, a través de la inscripción en el Programa Adolescencia del Gobierno de la CABA, una política orientada a promover la inclusión social a través de actividades culturales, deportivas, de ciencia y tecnología y de capacitación.

Para el análisis de los datos construidos, se siguieron los lineamientos generales de la teoría fundamentada, utilizando como auxiliar el software Atlas ti. Aplicando los criterios de parsimonia y de alcance, se codificó el corpus de datos, identificando y analizando categorías emergentes (STRAUSS; CORBIN, 2006).

\section{Geometria de las vulnerabilidades: entre trayectorias, vínculos y contextos}

$\mathrm{Al}$ indagar las prácticas y significaciones de jóvenes que realizaban prácticas artísticas y deportivas en barrios vulnerabilizados de la CABA, emergieron dos 
categorías centrales que expresaban tensiones entre mostrar-ocultar y salir-estar en sus biografías. En vínculo con estas categorías y las herramientas conceptuales, se identificaron dos pruebas como núcleos analizadores: la prueba de la relación con otros y la prueba de las movilidades.

En el despliegue de la prueba de las movilidades se presentó la tensión entre los deseos y motivaciones para salir de ciertos espacios y crear, ingresar y permanecer en otros. Y, a su vez, las posibilidades y regulaciones que ello suponía para los jóvenes en ocasiones también para sus familias -, para atravesar desde procesos migratorios y múltiples movilidades residenciales en busca de bienestar y oportunidades laborales, hasta desplazamientos cotidianos para cumplir con obligaciones diarias, como el estudio, el trabajo o el cuidado de otros, y sostener además, prácticas artísticas y deportivas.

En el caso de la prueba de la relación con otros, este desafío se ubicó en la experiencia de generar, sostener vínculos e integrarse en nuevos espacios sociales, evitando todo conflicto posible. Al momento de interactuar emergían sentires específicos que implicaban un trabajo significativo para los jóvenes sobre sus cuerpos y emociones. Dicho trabajo expresaba un proceso de mostrar-ocultar aquello considerado aceptable - ser relajado, positivo, alegre, flaco -, frente a sensibilidades y corporalidades indeseables como la tristeza, el sufrimiento, la timidez o la gordura. Para sostener relaciones significativas necesitaron movilizar soportes que facilitaran el tránsito entre lo que ocultaban y lo que mostraban donde sus cuerpos y emociones se volvieron modos de hacer frente a estas pruebas en un movimiento propio e interno, y a la vez, producido ante la co-presencia de otros.

Las pruebas sociales se distinguen analíticamente, sin embargo, refieren a procesos que se tensionan y vinculan entre sí. Su abordaje se orienta a la conformación de una cartografía "[...] capaz de describir a la sociedad y sus principales problemas, condensándola en un conjunto de grandes pruebas estructurales [...] que se deben enfrentar y en cuyo enfrentamiento se producen los individuos" (ARAUJO; MARTUCCELLI, 2012, p. 15). Identificar las fuerzas sociales que impulsan estos desafíos, implica comprender el modo permanente en que interactúan las condiciones estructurales y las vidas cotidianas.

Del análisis transversal de estas pruebas se reconocieron dimensiones comunes, así como en el tipo de soportes movilizados para enfrentarlas, que permitieron advertir coincidencias en el modo en que fueron experimentadas por los entrevistados. A 
partir de la conceptualización de vulnerabilidad que desarrollan François Delor y Michel Hubert (2000), así como los aportes de la Salud Colectiva brasilera en torno a este concepto (AYRES et al., 2008; AYRES; PAIVA; BUSCHALLA, 2012), y la noción de geometría de los cuerpos generada por Scribano (2007), se propone su vinculación a través de una nueva categoría analizadora del proceso de individuación de los jóvenes y sus experiencias sociales: la geometría de las vulnerabilidades.

Como señala Gabriela Vergara (2017, p. 11) el uso de la noción de geometrías “[...] como metáforas de lo social nos permiten comprender las formas y lugares en que se disponen los agentes sociales en tanto corporalidades en el espacio/tiempo social". Las posibilidades de desplazamiento como prácticas corporales en tanto energía social disponible producen luchas por su disponibilidad, su valoración y aceptación. Las diversas condiciones etarias, de género, de clase o étnicas, entre otras, provocan diferentes y desiguales distribuciones de esas energías sociales que conforman una particular geometría de los cuerpos por los que se ubican y des-ubican a los individuos en función de las relaciones de distancia y proximidad aceptadas socialmente. A su vez, supone considerar la gramática de las acciones, es decir, la disponibilidad que tienen los agentes de sus propias acciones y la capacidad de disponer de su propio cuerpo (SCRIBANO; AIMAR, 2012; VERGARA, 2017).

La noción de geometría permite, además, indagar las posibles intersecciones entre fenómenos que se presentan como si no tuvieran relación o conexión entre sí, advirtiendo sus posibles articulaciones y transformaciones (VERGARA, 2017). En ese sentido, su vinculación con el concepto de vulnerabilidad genera la posibilidad de visibilizar las principales tensiones en los procesos de individuación, a la luz de las distancias y proximidades que manifiestan las múltiples vulnerabilidades que atraviesan las biografías de los jóvenes. Asimismo, permite considerar el modo en que operan las desiguales condiciones de vida en la producción de desafíos, así como en las oportunidades y limitaciones para enfrentarlos.

El concepto de vulnerabilidad alude a la disponibilidad de soportes, así como a la susceptibilidad de individuos y grupos sociales frente a los padecimientos y sus posibilidades de enfrentarlos, haciendo visibles los procesos de fragilización no sólo económica, sino jurídica, social y política de ciertos individuos o grupos. Focaliza en las relaciones sociales y se distingue de la nociones epidemiológicas de riesgo individual, al considerar totalidades sociales complejas y dinámicas donde los aspectos individuales - en tanto intersubjetividades, los aspectos sociales -como 
contextos de esas interacciones y su regulación por valores y normatividades - y los aspectos programáticos - como formas institucionalizadas de tales interacciones -, se encuentran en tensión o articulación, superando así la clásica visión dicotómica que distingue lo individual y lo colectivo (AYRES et al., 2008; PECHENY, 2013; DI LEO; CAMAROTTI, 2015). En ese sentido, se consideran las vulnerabilidades, no como situaciones dadas, sino como procesos complejos y dinámicos en los que -a partir del análisis empírico- es posible distinguir tres niveles (DELOR; HUBERT, 2000):

- Trayectorias individuales: cada individuo atraviesa en su biografía distintos acontecimientos de manera particular, aun cuando sean experiencias compartidas con otros actores en un momento histórico determinado. De ahí, la posibilidad de interpretar las prácticas y elecciones de los individuos a partir de la manera en que se van posicionando en el transcurso de su vida.

- Vinculos e interacciones: concebir al individuo y sus prácticas implica considerarlo en su relación con otros, advirtiendo la intersección entre sus diferentes trayectorias. Por ello, es preciso identificar los tipos de interacciones - en tanto relaciones de poder - que se establecen entre los agentes.

- Contextos socio-institucionales: en tales relaciones - los momentos y modos en los que ocurren - operan diversas instituciones y normas sociales, políticas y culturales en un período socio-histórico determinado, favoreciendo u obstaculizando el acceso y uso de diferentes soportes o a la exposición a mayores o menores niveles de fragilización social y política.

Comprender la peculiar geometría de las vulnerabilidades en las que se despliegan las experiencias sociales de los jóvenes se vuelve posible al dar cuenta de la forma en que actúan articuladamente esos tres niveles en sus biografías. A continuación, se analizan los modos en que las vulnerabilidades "ponen distancia", y también se producen "en la proximidad".

\section{Vulnerabilidades que ponen distancia}

Las trayectorias personales y familiares de los entrevistados pusieron de manifiesto diferentes tipos de movilidades en sus biografías. Los procesos migratorios, en particular, se sucedieron en un contexto social y político-económico en el que las 
condiciones de vida en sus lugares de origen aparecen asociados a condiciones de empobrecimiento que impulsan la búsqueda de trabajo y una mejor calidad de vida en otras ciudades como estrategia familiar, aspecto característico de las migraciones en la región (BENENCIA, 2009; CERRUTTI, 2009; MIRANDA; CRAVINO; MARTÍ GARRO, 2012).

En las últimas décadas, las políticas públicas, los medios de comunicación y el sentido común en general han otorgado a las migraciones diferentes valoraciones. En el caso de las movilidades de aquéllos provenientes desde países limítrofes como Bolivia y Paraguay, o de las provincias del norte argentino - lugares de origen de gran parte de los entrevistados y sus familias - fueron vinculados a sentidos negativizantes que estigmatizaron a estos migrantes y los colocaron en el lugar de un "otro" indeseado. Fue especialmente durante los noventa - momento en que migran las familias de los entrevistados - la época en que, aún con avances en materia legislativa migratoria, eran culpabilizados por los altos niveles de desocupación y empobrecimiento en Argentina y sus derechos sociales y laborales, como el acceso a la salud y a la seguridad social, se veían limitados. Tales discursos, además, estaban acompañados por una fuerte desvalorización social de su condición étnica (HALPERN, 2005).

En los relatos de los jóvenes estos procesos migratorios, aun cuando ocurrieran en sus primeros años de vida o incluso antes de sus nacimientos, se señalan como puntos de inflexión significativos en sus itinerarios personales. Sin embargo, las condiciones contextuales que enmarcaron estas migraciones, vulnerabilizándolas, aparecen mayormente invisibilizadas en sus narrativas. Aunque reconocen dificultades en los lugares de origen, lo que se destaca es el esfuerzo de sus padres para trasladarse en pos de un futuro mejor, así como las habilidades que desarrollaron para enfrentar los desafíos que presentaban las ciudades receptoras.

La búsqueda de bienestar continúa en distintos momentos de sus biografías y produce nuevos desplazamientos, que llevan a poner en práctica diferentes estrategias habitacionales. ${ }^{4}$ En consecuencia, se producen múltiples movilidades residenciales vinculadas a la búsqueda de oportunidades educativas y laborales que se advierten como responsabilidades de sus padres y de los propios jóvenes.

Para acceder a los medios considerados legítimos de progreso social y económico, vinculados a la educación y al trabajo, "salir" de los barrios en los que residen en este caso, barrios populares como villas y asentamientos - se convirtió en una 
necesidad. Para los adultos se vuelve una forma de poner distancia entre lo apropiado y lo inapropiado, lo deseable y lo evitable para las vidas de estos jóvenes, que se encuentra en los límites entre el afuera y el adentro del barrio.

En la conformación de los barrios populares, particularmente en el caso de las villas y asentamientos, la delimitación del adentro y el afuera ha resultado un aspecto fundamental en la construcción de fronteras simbólicas. Tales barrios han sido estigmatizados al ser vinculados a la violencia y a la inseguridad. Aun cuando se construyen y re-construyen transformándose continuamente, tienden a no ser reconocidos como barrios y como parte de la dinámica urbana (CRAVINO, 2009; VITALE, 2009). Sin embargo, los aspectos de carencia y peligrosidad vinculados a ellos, de manera paradójica, son utilizados para fundamentar la ubicación de la población que los habita como potenciales "beneficiarios" de intervenciones estatales y la construcción de éstos como sujetos-objetos legítimos de las políticas sociales (CRAVINO, 2009). En el caso de los entrevistados, sus condiciones de vida vinculadas con los niveles de ingreso familiar, el tipo de vivienda en el que residen y la localización de los barrios en los que habitan, los ubicó en el lugar de posibles integrantes de los talleres ofrecidos por el Programa Adolescencia - del cual participó la mayoría de ellos - que se encuentra orientado a jóvenes que "integren hogares en situación de vulnerabilidad social" para promover su proceso de inclusión social a partir de las prácticas de expresión artística y las actividades físicas y deportivas.

Por otra parte, al dar cuenta de los obstáculos que experimentaron los jóvenes en sus interacciones en diferentes ámbitos - escuelas, universidades, boliches, espacios recreativos, etc. - la vinculación de dichas dificultades con sus condiciones de vida no era una dimensión que resaltaran en sus relatos. En cambio, percibían la necesidad de establecer acciones que, desde lo individual, les permitieran enfrentar los conflictos para presentarse en esos espacios y circular de manera adecuada, sin considerar los alcances y limitaciones para contar con soportes materiales y simbólicos.

Al reconstruir las significaciones y las vivencias corporales de los jóvenes ante sus posibilidades de transitar por el espacio urbano y circular por diferentes ámbitos, dentro y fuera del barrio, al nivel de sus interacciones y de sus trayectorias individuales, las nociones de cuerpo imagen, cuerpo piel y cuerpo movimiento (SCRIBANO, 2007; 2013), posibilitan aquí analizar y comprender las maneras en que operan las corporalidades y emociones en su sociabilidad. 
Todo individuo debe construir un cuerpo imagen que refiere al "cómo veo que me ven", donde el cuerpo aparece inscripto y situado socialmente según el modo en que es valorado. Así, en las experiencias de los jóvenes la percepción de sus propios cuerpos y emociones y la forma de adecuarse a las interacciones para "sentirse parte" en distintos ámbitos, estuvo vinculado a la conformación de una compostura en donde aparece simultáneamente el mostrar-ocultar lo que fuera considerado aceptable por los otros. En la tensión entre mostrar, no mostrar y observar a otros, se definían las posibilidades de éxito o fracaso para establecer relaciones que deseaban establecer y mantener, así como espacios, concebidos públicos o íntimos, que intentaban mantener integrados o separados.

En las interacciones cotidianas esto supuso - sin expresar diferencias significativas por género - tener que mostrarse como personas alegres, divertidas, donde la sonrisa en el rostro resultaba el principal rasgo enfatizado de una corporalidad disponible para la mirada de otros. Ello implicaba, asimismo, ocultar aquello que era percibido como debilidad asociada a los sentimientos de tristeza, sufrimiento o timidez. El cuerpo piel permite distinguir cómo los sentidos y las emociones que se viven desde lo individual, pasan a constituirse en sensibilidades sociales que naturalizan las formas de sentir-el-mundo.

En el contexto de las sociedades actuales se multiplican los medios para comunicar y se genera incluso una hipercomunicación (SCRIBANO, 2007). Sin embargo, paradójicamente no todos pueden ser vistos, contados u oídos. La vista apareció como uno de los sentidos prevalentes en las interacciones, enfatizando aquello que debe ser mostrado como una sonrisa o un cuerpo delgado y modelado, que no sólo posibilita ciertas relaciones con otros, sino que, al mismo tiempo, impone distancias con éstos. La mirada hacia sí mismos se traslada hacia los otros - junto con la escucha - como una forma de regular el modo en que se interactúa de modo precautorio y desconfiado.

Para los entrevistados ha resultado muy difícil expresar sus malestares y sentirse escuchados sin resultar juzgados. Esto ha provocado, a lo largo de sus vidas, que fuera casi imposible confiar en otros, ya sean otros jóvenes o adultos. En ese sentido, fue significativo el hecho de que procuraran ocultar aspectos tan relevantes en sus biografías como las situaciones de violencia familiar o las prácticas para adelgazar que causaban efectos negativos en su salud. En cambio, ha sido a través de sus capacidades personales que concibieron la posibilidad de atravesar los distintos retos 
en sus biografías, superando internamente tristezas, enojos, dificultades, sobre todo, para evitar conflictos con los otros.

La conformación, sostenimiento y reproducción del régimen neoliberal capitalista activan procesos que operan regulando las expectativas sociales y produciendo prácticas que se hacen cuerpo para evitar potenciales conflictos posibles de ser comprendidos a partir de la noción de mecanismos de soportabilidad social. En vinculación con estos mecanismos se generan dispositivos que regulan las sensaciones y producen ciertas percepciones que disponen las formas subjetivas de "apreciarse en el mundo". A partir de éstos se ponen en juego esquemas que no ocultan conflictos, sino que los desplazan produciendo, como consecuencia, que los procesos sociales sean sentidos como propios e individuales, invisibilizando las condiciones sociales que intervienen en su conformación (SCRIBANO, 2013).

Ahora bien, en las biografías de los jóvenes, las relaciones sociales suponen también un cuerpo en acción, un cuerpo movimiento, que permite advertir potencialidades y limitaciones para el hacer, diferenciando disposiciones corporales. En cuanto a la capacitación para el trabajo y la búsqueda de oportunidades laborales, en su formación como futuros trabajadores, los jóvenes son interpelados a incorporar ciertos valores para ser personas proactivas y emocional y corporalmente flexibles para insertarse en el mercado laboral.

Si bien se resaltan las habilidades personales, se invisibilizan las condiciones de vida y los soportes, como los antecedentes escolares y el capital social, e incluso ciertas características físico-corporales, a través de los cuales se ingresa a determinados puestos de trabajo (PÉREZ; DELEO; MASSI, 2013). Así, por ejemplo, el domicilio - en particular, residir en villas - opera como un obstáculo al momento de solicitar empleo. Frente a estas dificultades, para disminuir la impronta del lugar de residencia, los jóvenes refuerzan lo que se considera una buena apariencia. Para ello, utilizan estrategias para distanciarse de las imágenes asociadas a los jóvenes de sectores populares como sujetos peligrosos y desinteresados, adaptando su apariencia física - corte de pelo, tipo de vestimenta, forma de hablar - y su actitud frente a posibles empleadores. Situaciones similares son experimentadas también por quienes concurren a ámbitos universitarios. Allí se pretende que la presencia física o la actitud personal - ya sea en la vestimenta, la postura corporal y la confianza al hablar - que se muestra a compañeros y profesores se asimile a la de los demás y oculte los rasgos que, para los entrevistados, pueden no resultar adecuados en dichos contextos. 


\section{Vulnerabilidades en la proximidad}

Los procesos migratorios familiares de los entrevistados alternaron entre diferentes lugares de origen y lugares receptores, pero supusieron una permanencia más estable en barrios populares del AMBA. Los residentes de estos barrios han presentado de manera predominante, un origen migrante (MARGULIS; URRESTI; LEWIN, 2007). Las posibilidades de inserción residencial y laboral para los migrantes, en particular durante los noventas y los inicios del siglo XXI, se vieron limitados a ciertos destinos y a determinados núcleos de actividad caracterizados por la informalidad y la precariedad. La ubicación de esos cuerpos como productivos sigue una lógica mercantil donde ciertas energías corporales y sociales se encuentran más expuestas a la extracción y expropiación, en pos de intereses económicos y políticos del capitalismo global, donde los países del sur se ven más desfavorecidos en el proceso de absorción y disponibilidad de tales energías.

En el caso de los entrevistados, ya sea primero uno de sus padres o sus familias completas, se asentaron en estos barrios y desarrollaron actividades laborales que siguieron las tendencias generales de los rubros en los que se insertaban los migrantes regionales en esa época, donde el trabajo doméstico, el sector textil y de la construcción resultaron centrales (CERRUTTI, 2009; GADEA; BENENCIA; QUARANTA, 2009).

Los procesos de desplazamiento, aun ante las dificultades que impusieron, permitieron mantener en la distancia vínculos familiares, sostenidos, a su vez, por personas que actuaron como redes que facilitaron el ingreso y permanencia en estos nuevos territorios. La inserción en las nuevas ciudades, y en particular en tales barrios, fue valorado por los jóvenes. Si bien las condiciones de vida vulnerabilizadas en estas áreas aparecen de algún modo naturalizadas, en sus relatos se reconoce como la principal, e incluso, la única vía para sostenerse tras el proceso migratorio y para el acceso a una vivienda familiar.

No obstante, en dichos barrios también señalaron interacciones cotidianas que han sido experimentadas como situaciones de peligro. Frente a éstas, los jóvenes resaltaron el despliegue de estrategias personales para entrar y salir de los barrios y sostener distintas actividades en sus vidas cotidianas. Tales prácticas suponían estar atentos a quienes pudieran acercarse a ellos en la calle, establecer horarios para transitar o seleccionar algunos recorridos para moverse. Lo visual, especialmente, 
les permitía reconocer movimientos extraños, rostros o actitudes amenazantes, caminos más seguros; o por el contrario, recurrir a amigos o conocidos del barrio, que eran movilizados como soportes que brindaban cuidado y protección frente a situaciones que eran leídas como peligrosas. Los sentires vinculados a la confianza y a la desconfianza, sin embargo, como se señalara más arriba, no sólo aludieron al desplazamiento por los espacios públicos o al encuentro con personas desconocidas. Resultó altamente relevante el modo en que la construcción de esa confianza marcaba de manera sustantiva las interacciones de los jóvenes en todos sus espacios de sociabilidad, incluso los más íntimos.

En algunos casos la flexibilidad o rigidez para circular por el espacio público fue establecida por los adultos, quienes definiendo horarios y actividades, determinaban los desplazamientos cotidianos de los jóvenes. En ciertas condiciones aquéllos buscaban limitar sus salidas del barrio -especialmente en el caso de las mujerescon el fin de evitar "malas compañías" y como forma de protección ante los peligros que podían asociarse a estos espacios. Sin embargo, para realizar aquellas actividades que eran valoradas por los adultos -como la concurrencia a escuelas prestigiosas o la realización de actividades de formación como el estudio de idiomas fuera del barrioel tener que desplazarse entre distancias importantes sin compañía de adultos, no parece ser advertido por éstos como un problema para la seguridad de las movilidades cotidianas de los jóvenes.

Las prácticas cotidianas de los entrevistados reflejaron, no obstante, la potencia de un cuerpo movimiento, un cuerpo capaz de poner en acción movilidades que, simultáneamente, permitieran responder a esos límites que suponían las regulaciones al interior de la familia o a las normativas sociales acerca de las formas legitimadas de estudio y trabajo, y por otro lado, poner en práctica aquellas actividades deseadas y disfrutadas. Tener la disponibilidad de sus energías corporales, no sólo supuso poder circular libremente o poner sus cuerpos en movimiento. Como fue posible advertir en los relatos, también implicó el desarrollo de la capacidad de agencia en los jóvenes a partir de sus elecciones de permanecer en espacios-tiempos deseados, elegidos para 'escapar', para relajarse y para encontrarse con quienes han resultado significativos para ellos, como sus compañeros de circo, de futbol, de danza, o incluso también, los referentes y profesores de tales actividades.

Aun frente al desafío de tener que presentarse ante otros, la posibilidad de transitar por esos espacios y generar vínculos con personas que se volvieron relevantes en el 
marco de las actividades artísticas y deportivas, permitió a algunos de estos jóvenes reconocerlos como soportes simbólicos que no habían encontrado en otros ámbitos. En estos espacios de actividades, sobre todo los que se desarrollaban en el Circo, se pusieron de manifiesto tensiones entre la vergüenza que provocaba el temor de mostrarse ante otros, a hacer el ridículo o a equivocarse y las ganas de presentar aquello a lo cual habían dedicado tiempo y esfuerzo, que había generado placer y que al ser compartido con otros, se habían transformado en momentos significativos en sus biografías. En particular, los grupos con quienes se compartían prácticas artísticas como podían ser los compañeros del circo, los de un grupo de baile hiphop que se constituyó en un parque y hasta los skaters con quienes se comparte una rampa, permitían no sólo atravesar diferentes retos, sino también producir nuevos espacios-temporalidades producto de prácticas intersticiales (SCRIBANO, 2011) en los que fuera posible disfrutar, compartir placeres y relajarse ante la posibilidad de detenerse en ese encuentro con otros.

\section{Reflexiones finales}

En este trabajo se indagaron las articulaciones y tensiones entre las dimensiones singulares y estructurales que configuran las biografías de jóvenes de barrios populares en la CABA, a partir de la identificación de dos pruebas significativas en sus procesos de individuación: la prueba de las movilidades y la prueba de la relación con otros. Su análisis puso de manifiesto desigualdades en la distribución de las vulnerabilidades entre distintos grupos sociales y los consecuentes desafíos que éstas provocan.

A su vez, las posibilidades de afrontarlas exitosamente expresaron una particular geometría, que permite entrever el modo en que se manifiestan y se profundizan tales vulnerabilidades en el marco de relaciones de distancia y proximidad. En ella, se han ubicado a los jóvenes de sectores populares y a sus familias en ciertos lugares físicos y simbólicos, determinando por dónde pueden desplazarse y permanecer o qué corporalidades y emociones deben expresar. Esto ha supuesto un trabajo sobre sí mismos que es percibido por los entrevistados como esfuerzos y habilidades personales que invisibilizan las desiguales oportunidades existentes para el acceso a la vivienda, al trabajo, a la educación, a las prácticas artísticas y de recreación o al uso y circulación por el espacio público. 
La posibilidad de experimentar bienestar y lograr un futuro exitoso se habilita así a partir de movilidades migratorias y residenciales que establecen distancias entre los contextos de empobrecimiento, inseguridad y violencia asociados a los lugares que han habitado estos jóvenes y sus familias, frente a una imagen de prosperidad que habilita el residir y circular por zonas centrales de la CABA: escuelas prestigiosas, futuros universitarios, amistades adecuadas o trabajos de calidad.

Los jóvenes reconocen y valoran que tanto ellos como sus familias han realizado esfuerzos significativos y han utilizado habilidades personales para sostenerse en dicho contexto. Se muestran proactivos, alegres y delgados. Ocultan formas de hablar o de vestir habituales. Observan detenidamente el modo en que los otros actúan. Ya sea para protegerse o para ser semejante a aquéllos, los jóvenes procuran convertirse en integrantes válidos de los espacios por los que transitan y de los cuales desean ser parte.

Las vulnerabilidades se advierten también en la cercanía, en la proximidad con otros. Los barrios populares donde consiguen residir los jóvenes y sus familias tras los procesos migratorios se reconocen como lugares donde, aun en condiciones de precariedad, se torna posible adquirir una vivienda propia. No obstante, los barrios devienen, al mismo tiempo, espacios de peligrosidad. Desplazarse implica incorporar estrategias de cuidado como horarios o caminos elegidos, o la decisión de recurrir a otros como soportes y, de este modo, evitar contactos indeseados.

Aun cuando se advierten tales sensaciones de desconfianza y precaución continúas, los jóvenes cuentan con la posibilidad de vivenciar otras experiencias que se vuelven significativas y habilitan nuevos soportes para enfrentar estos retos cotidianos. Desde personas que facilitan una vivienda al asentarse en una nueva ciudad, el sostenimiento de contactos con familiares a la distancia o la creación de vínculos en espacios que favorecen la emergencia de solidaridades, placeres, encuentros, si bien no se diluyen las vulnerabilidades en su cotidianeidad, se generan rupturas y movimientos que ponen en cuestión aquellos lugares en los que son habitualmente ubicados en función de una posición social asociada a la carencia, la dificultad, la imposibilidad.

El análisis de las geometrías facilitó, de este modo, dar cuenta de la manera en que se crean e imponen distancias y marcas diferencialmente valoradas; se establecen desigualdades en la disposición de los cuerpos/emociones y las potenciales (in) movilidades. No obstante, también pudieron destacarse las posibilidades que generan ciertos espacios y vínculos, como los propuestos desde el Circo, para la generación de 
soportes que establecen resistencias y fugas a esas limitaciones, constituyéndose en experiencias relevantes en las biografías de estos jóvenes.

\section{Referencias}

ARAUJO, K.; MARTUCCELlI, D. Desafíos comunes. Retratos de la sociedad chilena y sus individuos. Santiago de Chile: LOM, 2012. 264p.

AYRES, J. R. M. C. et al. El concepto de vulnerabilidad y las prácticas de salud: nuevas perspectivas y desafíos. In: CZERESNIA, D.; FREITAS, M. C. (Org.) Promoción de la salud. Conceptos, reflexiones y tendencias. Buenos Aires: Lugar, 2008, p. 135- 162.

AYRES, J. R. C. M.; PAIVA, V.; BUCHALLA, C. M. Direitos humanos e vulnerabilidade na prevenção e promoção da saúde: uma introdução. In: PAIVA, V.; BUCHALLA, C. M.; AYRES, J. R. C. M. (Coord.) Vulnerabilidade e direitos humanos. Prevenção e promoção da saúde. Libro I. Da doença à cidadania. Curitiba: Juruá, 2012, p. 71-94.

BENENCIA, R. El infierno del trabajo esclavo. La contracara de las 'exitosas' economías étnicas. Avá. Revista de Antropología, n. 15, p. 1-32, 2009.

CERRUTTI, M. Gender and Intra-regional regional migration in South America. Buenos Aires: Human Development Research Papers. United Nations Development Programme, 2009, 68p.

CRAVINO, M. C. Vivir en la villa. Relatos, trayectorias y estrategias habitacionales. Buenos Aires: Universidad Nacional de General Sarmiento, 2009. 238p.

CRESSWELL, T. Towards a Politics of Mobility. Environment and planning. D, Society and space, v. 1, n. 28, p. 17-31, 2010.

DELOR, F.; HUBERT, M. Revisiting the concept of "vulnerability". Social Science \& Medicine, n. 50, p. 1557-1570, 2000.

DI LEO, P. F.; CAMAROTTI, A. C. (Eds.) "Quiero escribir mi historia". Vidas de jóvenes en barrios populares. Buenos Aires: Biblos, 2013. 320p.

DI LEO, P. F.; CAMAROTTI, A. C. (Dirs.). Individuación y reconocimiento. Experiencias de jóvenes en la sociedad actual. Buenos Aires: Teseo, 2015. 370p.

DI VIRGILIO, M.;GIL y DE ANSO, M. Estrategias habitacionales de familias de sectores populares y medios residentes en el área metropolitana de Buenos Aires (Argentina). Revista de Estudios Sociales, n. 44, p. 158-170, 2012.

GADEA, E.; BENENCIA, R.; QUARANTA, G. Bolivianos en Argentina y en España: De la migración tradicional a las nuevas rutas. AREAS. Revista Internacional de Ciencias Sociales, n. 28, p. 30-43, 2009. 
HALPERN, G. Neoliberalismo y migración: paraguayos en Argentina en los noventa. Política y Cultura, n. 23, p. 67-82, 2005.

LECLERC-OLIVE, M. Temporalidades de la experiencia. Las biografías y sus acontecimientos. Iberofórum. Revista de Ciencias Sociales de la Universidad Iberoamericana, n. 8, p. 1-39, 2009.

MARGULIS, M.; URRESTI, M.; LEWIN, H. Familia, hábitat y sexualidad en Buenos Aires. Investigaciones desde la dimensión cultural. Buenos Aires: Biblos Sociedad, 2007. 318p.

MARTUCCELLI, D. Cambio de rumbo. La sociedad a escala del individuo. Santiago de Chile: LOM, 2007. 242p.

. Lecciones de sociología del individuo. Pontificia Universidad Católica de Perú: Lima, 2006. 169p.

MIRANDA, A.; CRAVINO, M. C.; MARTÍ GARRO, S. Transiciones juveniles de migrantes paraguayos/as en la argentina: condiciones de vida y vigencia de las redes. Última Década, n. 37, p. 11-39, 2012.

PECHENY, M. Desigualdades Estructurales, Salud de Jóvenes LGBT y Lagunas de Conocimiento: ¿Qué Sabemos y qué Preguntamos? Temas em Psicologia, v. 2, n. 21, p. 961-972, 2013.

PÉREZ, P.; DELEO, C.; FERNÁNDEZ MASSI, M. Desigualdades sociales en trayectorias laborales de jóvenes en la Argentina, Revista Latinoamericana de Población, v. 7, n. 13, p. 6189, 2013.

SCRIBANO, A. A modo de epílogo. ¿Por qué una mirada sociológica de los cuerpos y las emociones? In: FIGARI, C.; SCRIBANO, A. (Comp.), Cuerpo(s), Subjetividad(es) y Conflicto(s). Hacia una sociología de los cuerpos y las emociones desde Latinoamérica. Buenos Aires: CICCUSCLACSO, p. 141-151, 2009.

SCRIBANO, A. El proceso de investigación social cualitativo. Buenos Aires: Prometeo Libros, 2008. 304p.

- Hacia unas ciencias sociales del Sur. Pensar las prácticas autonómicas entre el imperialismo, la dependencia y el colonialismo. Revista Pensamiento Plural, n. 8, p. 11-36, 2011. - ¡Salud, dinero y amor! Narraciones de estudiantes universitarios sobre el cuerpo y la salud. In: - (Comp), Policromía corporal. Cuerpos, grafías y sociedad. Córdoba: Universitas y UNC-Univ. de Guadalajara, 2007, p. 97-123.

- Sociología de los cuerpos/emociones. Revista Latinoamericana de Estudios sobre Cuerpos, Emociones y Sociedad-RELACES, n. 10, 93-113, 2013.

- Teorias sociales del Sur: Una mirada post-independentista. Buenos Aires: ESEditora/E-

Book Córdoba: Universitas - Editorial Científica Universitaria, 2012. 254p. 
SCRIBANO, A.; AIMAR, L. Presentación: Geometrías de los cuerpos. Distancias, proximidades y sensibilidades. Cuerpos, emociones y sociedad, n. 9, v. 4, p. 28-37, 2012.

STRAUSS, A.; CORBIN, J. Bases de la investigación cualitativa. Técnicas y procedimientos para desarrollar la teoría fundamentada. Bogotá: CONTUS, Universidad de Antioquía, 2006. 340p.

TAPIA, Silvia. Cuerpos, emociones e individuación: un análisis de las experiencias de jóvenes que realizan prácticas artísticas y deportivas en barrios populares de la Ciudad Autónoma de Buenos Aires. Tesis de doctorado inédita. Argentina: Universidad de Buenos Aires, 2016. 252p.

VERGARA, G. Distancias, formas y procesos: a modo de introducción. In: Vergara, G.; De Sena, A. (Comps.) Geometrías sociales. CABA: Estudios Sociológicos Editora, 2017, p. 9-26.

VITALE, P. La ley y la trama: Villas y política pública en la Ciudad. Apuntes sobre la trayectoria del Programa de Radicación, Integración y Transformación de Villas y Núcleos Habitacionales Transitorios. $5^{\circ}$ Jornadas de Jóvenes Investigadores. IIGG/Fac. Ciencias Sociales/UBA, 2009.

\section{Nota}

${ }^{1}$ Título de tesis: "Cuerpos, emociones e individuación: un análisis de las experiencias de jóvenes que realizan prácticas artísticas y deportivas en barrios populares de la Ciudad Autónoma de Buenos Aires" (2016). Doctorado de la Universidad de Buenos Aires en Ciencias Sociales. Financiada por: Consejo de Investigaciones Científicas y Técnicas (CONICET), Argentina. Director: Dr. Pablo F. Di Leo. Co-director: Dr. Danilo Martuccelli.

${ }^{2}$ Teniendo conciencia de la orientación androcéntrica del español, utilizamos el género masculino en los plurales sólo para facilitar la lectura.

3 "El Circo" es el modo en que nombran este espacio los que participan cotidianamente en la institución. Esta organización social funciona desde el año 1998. Allí se dictan talleres artísticos gratuitos en diferentes barrios de la CABA, especialmente en los más vulnerabilizados, con el propósito contribuir a la transformación personal, comunitaria y social a través del arte.

${ }^{4}$ Las estrategias habitacionales refieren a las lógicas, decisiones y objetivos, así como la capacidad de movilizar recursos, de acuerdo a las condiciones sociales, económicas y culturales de los individuos y los hogares, para establecer diferentes recorridos residenciales en la búsqueda por el acceso a la vivienda (DI VIRGILIO; GIL Y DE ANSO, 2012). 


\section{Abstract}

Vulnerabilities, mobilities and corporalities in young people who perform practices in arts and sports in popular neighborhood of Buenos Aires City

The paper presents results of a doctoral thesis, which analyzed the experiences of young people from popular neighbourhoods who perform practices in arts and sports in the Autonomous City of Buenos Aires, investigating the linkages between bodies, emotions and space mobilities in their processes of individuation. The analysis focuses on the reading of the tensions and articulations between the social challenges that these young people face and the supports to pass through them, from the use of the category of geometry of the vulnerabilities, arising from the articulation of the notions of vulnerability and geometry of bodies. For the construction of the empirical data, semi-structured interviews, biographical accounts, and participationobservation were conducted in a social organization that offers free workshops in these areas. This approach made it possible to notice the dynamics and multidimensionality of vulnerability processes - where the linkages among bodies-emotion-mobilities revealed particular and novel forms of these processes and supports to face them - that cross over the experiences of young people from popular neighborhoods.

> Keywords: youths; vulnerabilities; mobilities; bodies; emotions. 


\section{Resumo}

\section{Vulnerabilidades, mobilidades e} corporalidades em jovens que realizam práticas artísticas e esportivas em bairros populares da Cidade de Buenos Aires $\mathrm{O}$ artigo apresenta os resultados de tese de doutorado na qual foram analisadas as experiências de jovens de bairros populares que realizam práticas artísticas e esportivas na Cidade Autônoma de Buenos Aires, procurando as ligaçóes entre corpos, emoçóes e mobilidades espaciais em seus processos de individuação. Aqui a análise centra-se na leitura das tensōes e articulaçôes entre os testes sociais que enfrentam esses jovens, e os suportes para atravessálos, a partir da utilização da categoria de geometria das vulnerabilidades, surgida da articulaçâo das noçóes de vulnerabilidade e geometria dos corpos. Para a construção dos dados empíricos, realizaramse entrevistas semiestruturadas, relatos biográficos $\mathrm{e}$ participação-observação em uma organização social que oferece cursos gratuitos nesses bairros. Esta abordagem permitiu notar a dinâmica e multidimensionalidade dos processos de vulnerabilidade -, em que as relações entre corpos-emoçóes-mobilidades manifestaram formas particulares e inovadoras desses processos e dos suportes para lidar com eles - que atravessam as experiências de jovens de bairros populares.

> Palavras-chave: juventudes; vulnerabilidades; mobilidades; corpos; emoções. 